\title{
SPECTRUM OF MAGNETIC RESONANCE IMAGING ABNORMALITIES OF HYPOXIC ISCHEMIC ENCEPHALOPATHY IN PRETERM AND FULL-TERM PATIENTS AT TERTIARY CARE HOSPITAL IN INDIA
}

\author{
Daisy Gupta1, Deepak Rana ${ }^{2}$
}

${ }^{1}$ Assistant Professor, Department of Radio-diagnosis, GMC Amritsar, Punjab, India.

2Junior Resident, Department of Radio-diagnosis, GMC Amritsar, Punjab, India.

\section{BACKGROUND}

ABSTRACT

Hypoxic Ischemic Encephalopathy (HIE) is one of the most common neurological abnormalities causing neonatal mortality and severe neurological deficits in children. HIE also constitutes an important diagnostic dilemma in both full term and prematurely born infants. Many times HIE goes unnoticed in early stages, only to present in later stages of life as a severe neurological deficit in children. The various patterns seen in HIE depend on the duration and severity of hypoxia, and brain maturation level. Therefore, imaging findings of HIE highly depend upon the timing of imaging. MRI plays a vital role in early detection of the various spectrum of HIE. It not only helps radiologist to the right path of diagnosis but also guides paediatrician in determining the prognosis of the development of the infant.

Aims and Objectives- To retrospectively study the spectrum and various patterns in which HIE affects preterm and full-term infants and to evaluate the common presentations on MRI.

\section{MATERIALS AND METHODS}

This Retrospective descriptive study was done on 50 patients, diagnosed with HIE, visiting the Department of Radio-diagnosis, GMC, Amritsar for MRI examination on 1.5 Tesla Siemens machine. Various patterns by which HIE affects preterm and full-term infants were studied.

\section{RESULTS}

Our study included 50 patients diagnosed with HIE. 18 (36\%) of these patients were pre-terms and 32 (64\%) patients were full term. $35(70 \%)$ of these were males and $15(30 \%)$ were females. The most common clinical presentation was developmental delay. Most common MRI finding in preterm patients was periventricular leukomalacia and in full term patients was T2 and FLAIR periventricular and subcortical white matter hyperintensities.

\section{CONCLUSION}

MRI has become the primary imaging modality in patients suffering from HIE and plays a pivotal role in identification, localization and characterization of the underlying pattern of disease in affected patients. The combination of various sequences in MRI help in early diagnosis of HIE lesions and are highly needed early management of the disease.

\section{KEY WORDS}

Magnetic Resonance Imaging, Hypoxic Ischemic Encephalopathy, Preterm, Term.

HOW TO CITE THIS ARTICLE: Gupta D, Rana D. Spectrum of magnetic resonance imaging abnormalities of hypoxic ischemic encephalopathy in preterm and full-term patients at tertiary care hospital in India. J. Evolution Med. Dent. Sci. 2018;7(51):54605465, DOI: $10.14260 /$ jemds/2018/1208

\section{BACKGROUND}

Hypoxia refers to decreased oxygen supply and ischemia due to decreased blood supply to the tissue, which leads to the resultant damage of brain tissue. If an episode of hypoxiaischemia is severe enough to damage the brain, it leads to a neonatal encephalopathy within 12 to 36 hours known as hypoxic-ischemic encephalopathy (HIE). ${ }^{1}$ HIE results due to a combination of hypoxia, hypercarbia and metabolic acidosis. This may be a consequence of occlusion of umbilical vessels or interference with placental perfusion in foetal life or due to lack of effective breathing after birth.

'Financial or Other Competing Interest': None.

Submission 28-08-2018, Peer Review 01-12-2018,

Acceptance 08-12-2018, Published 17-12-2018.

Corresponding Author:

Dr. Daisy Gupta,

Assistant Professor,

Department of Radio-diagnosis,

GMC Amritsar, Punjab, India.

E-mail: daisygupta23@gmail.com

DOI: $10.14260 /$ jemds $/ 2018 / 1208$
Insufficient cerebral blood flow and hypoxia leads to loss of normal cerebral autoregulation. This eventually leads to a series of events leading to injury to the brain tissues with clinical manifestations. The incidence of HIE is 2.5 per 1000 in term live births and approximately 7 per 1000 preterm births. $^{2}$

Almost $40 \%$ of deaths under the age of 5 years in children occur in the neonatal period. Reported global totals of neonatal deaths due to non-specific conditions of HIE vary from 0.7 million to 1.6 million per year. Mortality rate in children with severe hypoxia is $25-50 \%$. Incidence of prenatal asphyxia is approximately $3.3 \%$ in India. It is most commonly related to gestational age and birth weight. ${ }^{3}$ Hypoxic-ischemic injury results in $23 \%$ of world's neonatal deaths and causes permanent neurological deficits in $25 \%$ of the affected term neonates. ${ }^{4}$ The clinical diagnosis of HIE is based on evidence of foetal distress, low umbilical cord $\mathrm{pH}$ of $<7.1$ (acidosis), a poor Apgar score (0-3) at $5 \mathrm{~min}$, necessity for resuscitation, neurological abnormalities like seizure, coma, hypotonia and multiorgan dysfunction. ${ }^{2}$ HIE leads to 
dangerous and permanent damages, making it a major burden for the patient, family and the society. Thus, it is very important to identify, evaluate and analyse the effects of HIE on the brain so that early and better therapeutic strategies can be planned to decrease damage to the brain in newborns and provide them a better prognosis in the future.

Findings in HIE are highly variable and depend on brain maturity, severity and duration of asphyxia, as well as timing of imaging studies. Pattern of injury in preterm neonates ( $<37$ weeks gestational age) is distinct from that of the full term neonates ( $\geq 37$ weeks gestational age). ${ }^{5}$ Treatment is primarily supportive and aims at limiting the extent of brain injury. MRI allows to reveal a number of lesions associated with HIE, even very subtle lesions that are invisible on the ultrasound scans like cortico-subcortical lesions within the central region, bilateral parasagittal lesions and lesions in brain stem, thalamus, cerebellum and hippocampus. ${ }^{6}$ The role of MRI is in excluding structural anomalies and mainly in assessing the extent and nature of injury. Thereby, it helps in early diagnosis, prediction of clinical consequences and planning neurodevelopmental therapy.

\section{MATERIALS AND METHODS}

This Retrospective descriptive study was done on 50 patients diagnosed with HIE on 1.5 Tesla Siemens MAGNETOM Aera machine, who visited radiology department of GMC, Amritsar over a period of one year from April 2017 to March 2018. The study included all the patients of paediatric age group (Birth to 15 years) referred for MRI with suspicion of perinatal hypoxic injury. Various patterns by which HIE affects preterm and full-term infants were studied. It included neonates with clinical signs including seizures, hypotonia, bradycardia, poor feeding and a depressed level of consciousness. Developmental delay, cerebral palsy, seizures, hemiplegia/ paraplegia/quadriplegia were the criteria used in children for performing the MRI.

$\mathrm{T} 1$ and $\mathrm{T} 2$ Weighted images in axial plane, T2WI in coronal and sagittal planes, Diffusion Weighted images (DWI) in axial plane with apparent diffusion coefficient (ADC) map generation, Susceptibility Weighted images, and fluid attenuated inversion recovery (FLAIR) in axial plane were the key protocol employed. 3D sequences like MPRAGE and T2 SPC were used wherever required.

The standard MR sequences used in an adult brain were optimized for use in neonates. This is because of the higher water content and lower protein and lipid contents of neonatal brain. This was achieved by increasing the repetition time (TR) of both $\mathrm{T} 1$ and $\mathrm{T} 2$ weighted images. For T1WI, the standard TR (400 ms) was increased to $800 \mathrm{~ms}$, and for T2WI, the standard TR (4000 $\mathrm{ms})$ was increased to $6500 \mathrm{~ms}^{7}$

Axial T1WI is ideal for detecting myelination and subacute haemorrhage. Axial T2WI provides good contrast between gray and white matter and is useful in delineating white matter signal abnormalities. Gradient echo $\mathrm{T} 2 *$ or susceptibility WI (SWI) is ideal for demonstrating haemorrhage and distinguishing it from ischemic gray matter lesions and astrogliosis. DWI with ADC maps between 24 hours and 7 days of life is more sensitive for the detection of cytotoxic oedema than conventional T1 or T2WI. The ischemic areas show restricted diffusion in this time period, which is manifested as bright signal on DWI and reduced signal intensity on ADC maps. DWI abnormalities generally peak 3-5 days after the insult and subsequently normalize despite the tissue injury. Early DWI is excellent for the detection of white matter injury. ${ }^{2}$

The FLAIR sequence is useful for perception of abnormally increased T2 signal consistent with glial tissue and cystic lesions. Once myelination has occurred, the sequence is particularly useful for demonstrating periventricular and cortical gliosis. ${ }^{8}$

In general, DWI is most useful in the first week of life and conventional $\mathrm{T} 1$ and $\mathrm{T} 2 \mathrm{WI}$ are most diagnostically useful from the second week onwards. The recommended timing for MRI is between 5 and 14 days from birth. ${ }^{9}$

\section{RESULTS}

\section{Sex Wise Distribution}

Out of 50 patients, 35 were males and 15 were females as seen in the chart below. Thus, males were more commonly affected than females in this study with a sex ratio of 2.3:1.

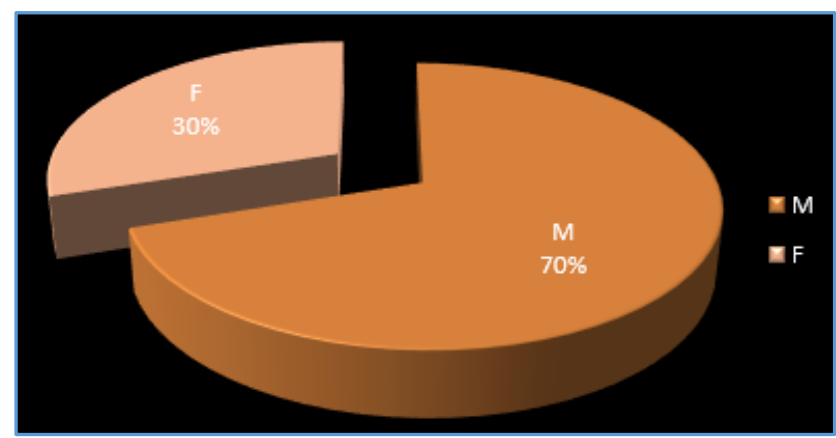

Figure 1. Sex Distribution of Patients

\section{Age Wise Distribution}

The age of patients ranged from 0 years to 15 years. The maximum pathologies were seen in the patients who were in the age group of 1 to 5 years as shown in following graphical presentation

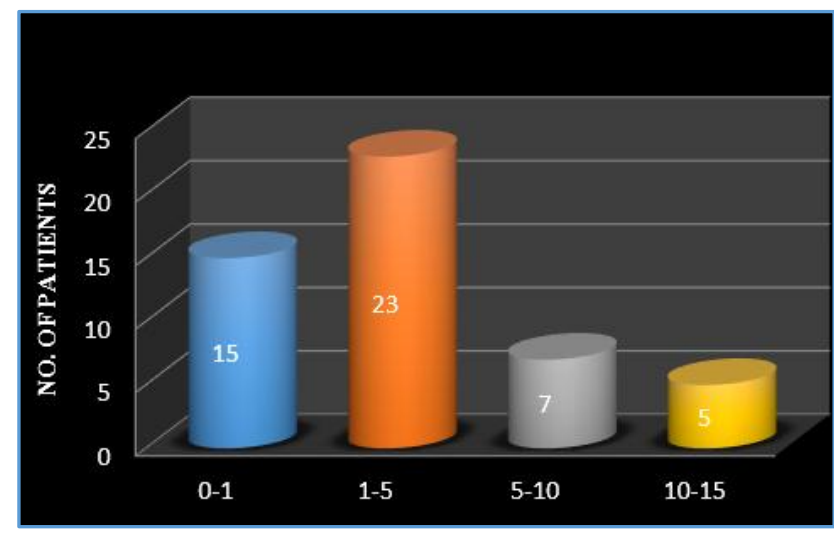

Figure 2. Age Wise Distribution of Patients

\section{Gestational Age Wise Distribution}

In our case study, 18 patients had a history of preterm delivery and 32 patients were term neonates. So, term patients were most commonly affected in our study. 


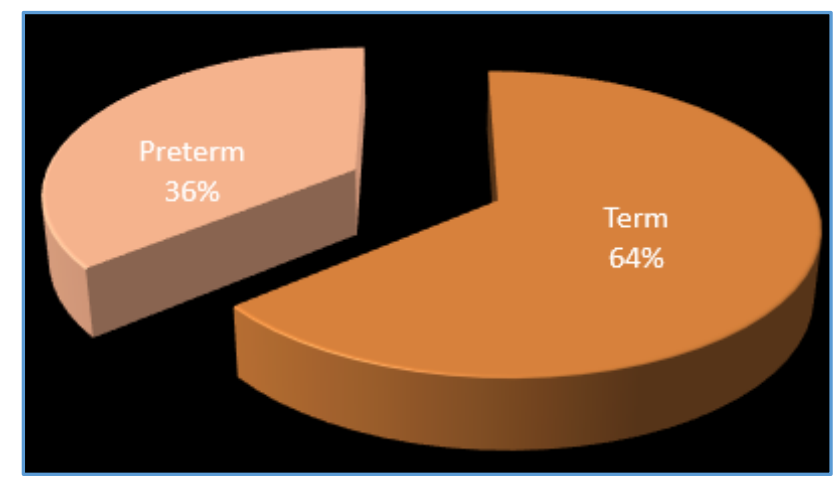

Figure 3. Distribution of Patients according to Gestational Age

\section{Imaging Findings in Term Neonates}

32 cases of term neonates who had suffered perinatal hypoxia underwent MRI. Out of them, 25 (78.1\%) showed T2 and FLAIR white matter hyperintensities, which was the most common abnormality found. Other commonly seen findings were cerebral atrophy, delayed myelination, encephalomalacia, corpus callosum thinning and acute infarcts.

\begin{tabular}{|c|c|c|c|}
\hline $\begin{array}{c}\text { Sr. } \\
\text { No. }\end{array}$ & MRI Findings & $\begin{array}{c}\text { No. of } \\
\text { Cases }\end{array}$ & (\%) \\
\hline 1 & T2 \& FLAIR WM Hyperintensities & 25 & 78.1 \\
\hline 2 & Cerebral Atrophy & 20 & 62.5 \\
\hline 3 & Encephalomalacia & 15 & 46.8 \\
\hline 4 & Delayed Myelination & 15 & 46.8 \\
\hline 5 & Corpus Callosum Thinning & 13 & 40.6 \\
\hline 6 & Basal Ganglia and Thalami & 8 & 25 \\
\hline 7 & Acute Infarcts & 3 & 1.2 \\
\hline 8 & Peri Rolandic Areas Hyperintensities & 2 & 6.25 \\
\hline \multicolumn{4}{|c}{ Table 1. MRI findings in HIE in Term Neonates (n=32) } \\
\hline
\end{tabular}

\section{Imaging Findings in Preterm Neonates}

18 cases of preterm neonates who suffered perinatal hypoxia were evaluated in this study by MRI. The most common findings were cerebral atrophy 11 (61.1\%) and periventricular leukomalacia in 10 (55.56\%). Other pathologies which were observed were corpus callosum thinning, delayed myelination, acute infarcts and germinal matrix haemorrhage.

\begin{tabular}{|c|c|c|c|}
\hline Sr. No. & MRI Findings & No. of Cases & $\mathbf{( \% )}$ \\
\hline 1 & Cerebral Atrophy & 11 & 61.1 \\
\hline 2 & Periventricular Leukomalacia & 10 & 55.56 \\
\hline 3 & Corpus Callosum Thinning & 9 & 50 \\
\hline 4 & Delayed Myelination & 8 & 44.45 \\
\hline 5 & Acute Infarcts & 3 & 16.67 \\
\hline 6 & Germinal Matrix Haemorrhage & 3 & 16.67 \\
\hline \multicolumn{3}{|c}{ Table 2. MRI findings in Preterm Neonates (n=18) } \\
\hline
\end{tabular}

\section{Distribution of Findings on MRI in Patients with Cerebral Palsy}

Out of 50 cases HIE, 8 cases presented with cerebral palsy. Out of these 8 patients, 3 (37.5\%) were preterm and 5 (62.5 $\%)$ cases were term. Cerebral atrophy was overall most common finding. Periventricular leukomalacia in preterm patients and hyperintense lesions on T2WI and FLAIR images in term infants, were the commonest findings. Other commonly seen findings were Corpus callosum thinning, cystic encephalomalacia and delayed myelination.

\begin{tabular}{|c|c|c|c|}
\hline Sr. No. & MRI Findings & No. of Cases & (\%) \\
\hline 1 & Cerebral Atrophy & 7 & 87.5 \\
\hline 2 & $\begin{array}{c}\text { T2 and FLAIR WM } \\
\text { hyperintensities }\end{array}$ & 6 & 75 \\
\hline 3 & Corpus Callosum Thinning & 5 & 62.5 \\
\hline 4 & Delayed myelination & 4 & 50 \\
\hline 5 & Cystic Encephalomalacia & 2 & 25 \\
\hline 6 & Periventricular Leukomalacia & 2 & 25 \\
\hline \multicolumn{3}{|c|}{ Table 3. Distribution of MRI findings in patients with } \\
cerebral palsy (n=8)
\end{tabular}

\section{DISCUSSION}

Hypoxic-ischemic encephalopathy (HIE) is a global issue especially in the developing countries like India. HIE in neonates is an important cause of mortality, morbidity and neurodevelopmental delay worldwide despite advances in the better understanding of pathophysiology of the new born. It can complicate into permanent brain damage. Now with the therapeutic advances effective treatment can be provided to the patients suffering from HIE if they are diagnosed early. Early identification and intervention is necessary to prevent the long term brain damage. Advances in MRI technique have made excellent progress over the last few years and now even subtle lesions can be identified.

\section{Normal MRI Appearances of Neonatal Brain}

Neonatal brain differs from adult brain in degrees of myelination and hence MR signal intensities of myelinated and unmyelinated white matter. In adults, myelination appears as high signal intensity on T1WI and low signal intensity on T2WI compared to the grey matter. Normally myelination starts by the $2^{\text {nd }}$ trimester and almost complete myelination occurs by the age of 2 years. So reversal of the normal white matter signal is seen in the neonatal brain. In normal neonates after 37 weeks of gestational age, Increased $\mathrm{T} 1$ signal intensity of myelination is seen in the posterior half of the posterior limb of the internal capsule and ventrolateral part of the thalamus. ${ }^{10}$

\section{Various Patterns of Brain Injury in Hypoxic-Ischemic Injury}

A hypoxic-ischemic event lasting for more than $10-15 \mathrm{~min}$ is enough to induce brain damage in the perinatal period. Basal ganglia and thalami, internal capsule, cortex, subcortical and periventricular white matter, and medial temporal lobe are the usual sites of brain injury in HIE. Various patterns of brain injury are seen depending upon level of brain maturity at the time of the insult and the severity and duration of the hypoxic-ischemic event.

\section{A) Mild to Moderate Brain Injury}

During mild to moderate hypoperfusion, autoregulation causes redistribution of blood flow to the metabolically hyperactive deep grey matter structures. This results in injury predominantly to the watershed zones of the cerebrum. The vascular supply of the brain changes with brain maturation. In the preterm brain, hypoperfusion results in a periventricular border zone of white matter injury. In the full term, hypoperfusion results in subcortical white matter and parasagittal cortical injury. 


\section{B) Severe Brain Injury}

In severe hypoperfusion, there is loss of autoregulation. The vulnerable regions are the deep grey matter and the early or actively myelinating fibers. In preterm neonates, severe asphyxia preferentially injures the thalami, dorsal brainstem, and anterior vermis with relative sparing of the basal ganglia and cortex. Severe asphyxia in term neonates causes injury to the posterior putamina, ventrolateral thalami, hippocampi and dorsal brainstem, and occasionally the sensorimotor cortex.

\begin{tabular}{|c|c|c|c|}
\hline $\begin{array}{l}\text { Sr. } \\
\text { No. }\end{array}$ & $\begin{array}{c}\text { Age (Post- } \\
\text { Conceptional in } \\
\text { Weeks) }\end{array}$ & $\begin{array}{c}\text { Mild to } \\
\text { Moderate } \\
\text { Injury }\end{array}$ & $\begin{array}{l}\text { Severe } \\
\text { Injury }\end{array}$ \\
\hline 1 & $\begin{array}{c}\text { Premature } \\
\text { Neonate } \\
(<37 \text { weeks })\end{array}$ & \begin{tabular}{|} 
Periventricul \\
ar white \\
matter and \\
Germinal \\
matrix \\
haemorrhag \\
es
\end{tabular} & $\begin{array}{l}\text { Thalamic, dorsal } \\
\text { brainstem and } \\
\text { anterior vermis } \\
\text { more frequently than } \\
\text { basal ganglia }\end{array}$ \\
\hline 2 & $\begin{array}{l}\text { Term Neonate } \\
\text { (>37 weeks) }\end{array}$ & \begin{tabular}{|c|} 
Subcortical \\
white matter \\
and \\
Parasagittal \\
watershed \\
region \\
\end{tabular} & \begin{tabular}{|c} 
Basal ganglia, \\
ventrolateral \\
thalami, \\
hippocampi and \\
dorsal brainstem, \\
lateral geniculate \\
nuclei and \\
sensorimotor cortex
\end{tabular} \\
\hline \multicolumn{4}{|c|}{ Table 4} \\
\hline
\end{tabular}

In this retrospective study, 50 patients of perinatal hypoxia presenting with various clinical signs symptoms were studied on MRI. Out of which, 35 (70\%) were males and $15(30 \%)$ were females with a sex ratio of 2.3:1. These findings are consistent with the study done by Lakhkar et al ${ }^{11}$ who showed a 3:1 male predominance in their study. In the study by Qureshi et al, $379.6 \%$ of cases were males and $20.4 \%$ were females.

In our study, maximum patients were of age group 1 to 5 years. In study done by Connolly et al, 12 the age group range taken into consideration was 1 to 24 years. Of them maximum patients were between the age group of 1 to 5 years. Thus, our findings correlate well this study.

In our study 18 (36\%) patients were preterms and 32 (64\%) had term delivery. These findings are in accordance with study performed by Yin et al. ${ }^{13}$ He carried out the study on 42 patients in whom 12 were premature $(28.5 \%)$ and 30 were full-term (71. 4\%). In another study done by Qureshi et $\mathrm{al}^{3}$ on 181 infants, out of 181 neonates $77.9 \%$ were full term, $19.1 \%$ were premature. In the study done by Khaldkar et al $^{14}$ on 100 patients, $36 \%$ patients were preterms and $64 \%$ had term. Thus, our findings correlate with all these studies.

We evaluated $32(64 \%)$ patients with a history of term delivery. The cortical and subcortical T2 and FLAIR hyperintensities were noted in 25 (78.1\%) cases. Cerebral atrophy was also common and found in 20 (62.5\%) cases. In patients presenting with acute symptoms, 4 (1.2\%) cases showed acute infarcts. Delayed myelination was seen in 15 $(46.8 \%)$ of the patients. Rutherford et $\mathrm{al}^{15}$ performed a study on patients with HIE and tried to find the correlation of MR findings with clinical outcome. He found cortical and subcortical T2 hyperintensities in $50 \%$ of the patients and cerebral atrophy in $50 \%$ of the cases. Basal ganglia infarction was observed in $18 \%$ cases and $6 \%$ cases showed insular infarcts. Delayed myelination was present in $50 \%$ of the infants.

We studied 18 (36\%) patients who were born with the history of preterm delivery. 11 (61.1\%) patients showed cerebral atrophy and $10 \quad(55.56 \%)$ cases showed periventricular leukomalacia (PVL) as the commonest findings. Corpus callosum thinning was observed in 9(50\%) cases and Delayed myelination was noted in $8(44.45 \%)$ out of 18 cases. Acute infarcts were seen in $3(16.67 \%)$ cases. Germinal matrix haemorrhage was diagnosed in 3(16.67\%) cases. In a study carried out by Gul Serdaroglu et al, 1689 children with PVL were evaluated. The aim of this study was to find out neurodevelopmental delay in children with periventricular leukomalacia. PVL was divided into 3 grades: grade I, unilateral or bilateral areas of periventricular hyperintensity; grade II, hyperintensities more than 3; grade III, hyperintense lesions more than 3 and ventricular wall irregularity; grade IV, diffuse PVL and ventricular dilatation. Thinning of the corpus callosum and cortical atrophy was identified respectively in $73 \%$ and $47.2 \%$ of the patients. Delayed myelination was noted in $14.3 \%$ cases. MRI was normal in $18 \%$ of the infants. Khaldkar et al ${ }^{14}$ studied 36 (28\%) patients who were born with the history of preterm delivery. Of these, $72.3 \%$ cases were found to be abnormal. $33.3 \%$ (12) cases showed periventricular leukomalacia and $33.3 \%$ (12) patients showed cerebral atrophy as the commonest findings. Delayed myelination and corpus callosum thinning was observed in $22.2 \%$ (8) cases. $27.7 \%$ cases did not show any significant abnormality on MRI. Acute infarcts were seen in $11.1 \%$ (4) cases. Cerebellar atrophy was present in $5.5 \%$ (2) cases. Germinal matrix haemorrhage was diagnosed in $5.5 \%$ (2) cases.

In our study, 8 patients presented with cerebral palsy. Out of these 8 cases, $3(37.5 \%)$ cases were preterm infants and $5(62.5 \%)$ were term patients. The commonest finding in term infants was T2WI and FLAIR hyperintense lesions and in preterm patients was periventricular leukomalacia. The most common overall finding in patients with cerebral palsy observed by us was cerebral atrophy 7 (87.5\%) followed by T2 and FLAIR hyperintensities 6 (75\%), corpus callosum thinning 5 (62.5\%), Delayed myelination 4 (50\%) and periventricular leukomalacia in $2(25 \%)$ of the cases. Yin et $\mathrm{al}^{15}$ performed a study of MRI findings in 42 patients, of which 8 were premature (38\%), 13 were full-terms (62\%). Periventricular leukomalacia was seen in $66.6 \%$. Cerebral atrophy was seen in $33.3 \%$ cases and $33.3 \%$ children did not demonstrate PVL. The other group he studied included 30 children who were term infants. The MRI findings demonstrated T2 hyperintensities as the most common finding seen in approximately $30 \%$ patients. It was followed by cerebral atrophy seen in $20 \%$ cases and other lesions in 20 $\%$ cases. Acute infarcts were noted in $13 \%$ of the patients. Delayed myelination was seen in $7 \%$ cases and corpus callosum thinning in $6 \%$. Truwit et al ${ }^{17}$ did a study on 40 patients with cerebral palsy. Of these, 11 were premature and 29 were term infants. Of the 11 patients born prematurely, MR revealed deep white matter loss, especially in the peritrigonal regions. $81 \%$ of the scans demonstrated thinning of the corpus callosum. No abnormalities were noted in the basal ganglia or thalami. $27 \%$ had diminished calibre of the brainstem. MR was done in 29 patients born at term, out of which $66 \%$ had diminished deep white matter. $6 \%$ had 
delayed myelination. In 55\%, the corpus callosum was thinned either focally (involving the posterior body and/or splenium) or diffusely. Ventricular abnormalities were seen in $41 \%$ with an irregular ventricular contour and ventricular enlargement and cerebral atrophy. Cortical thinning was seen in $10 \%$ term patients, one of whom had findings diagnostic of multicystic encephalomalacia. $20 \%$ cases had basal ganglia and/or thalami involvement. Overall our findings corroborate with both the studies.

Case 1

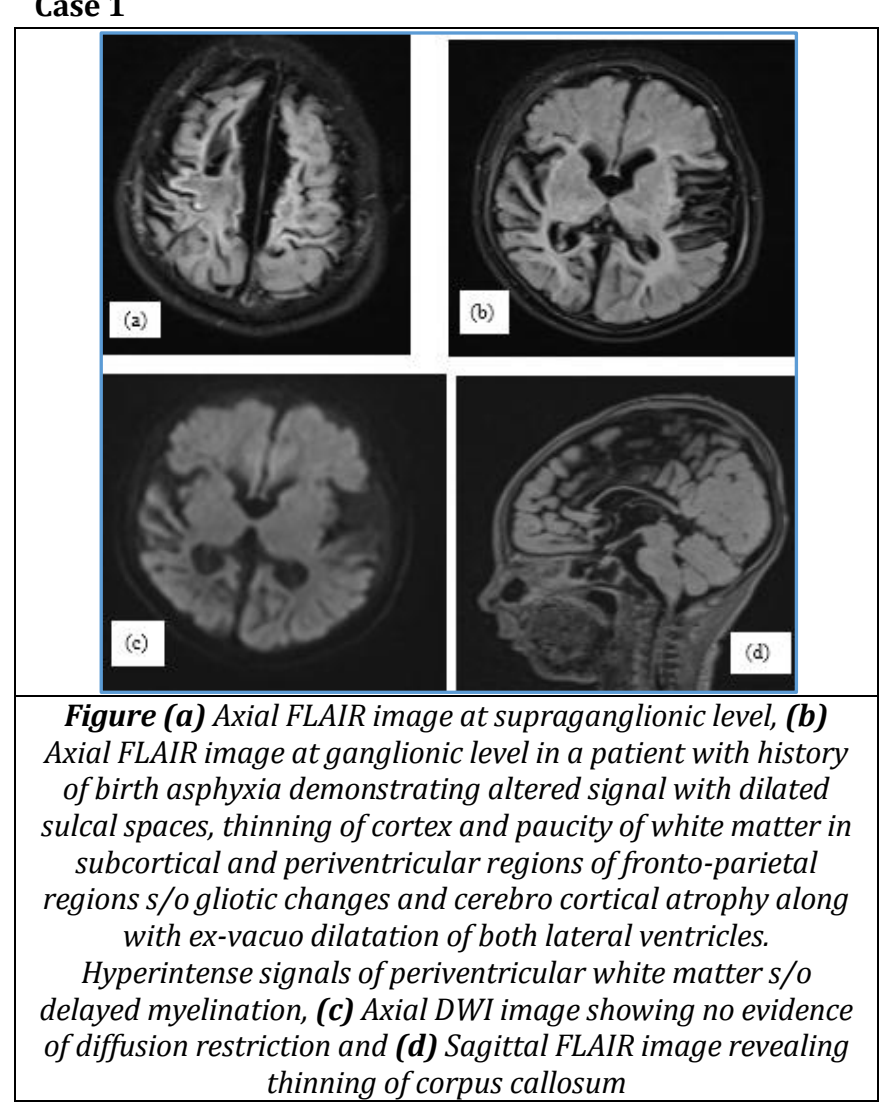

\section{Case 2}
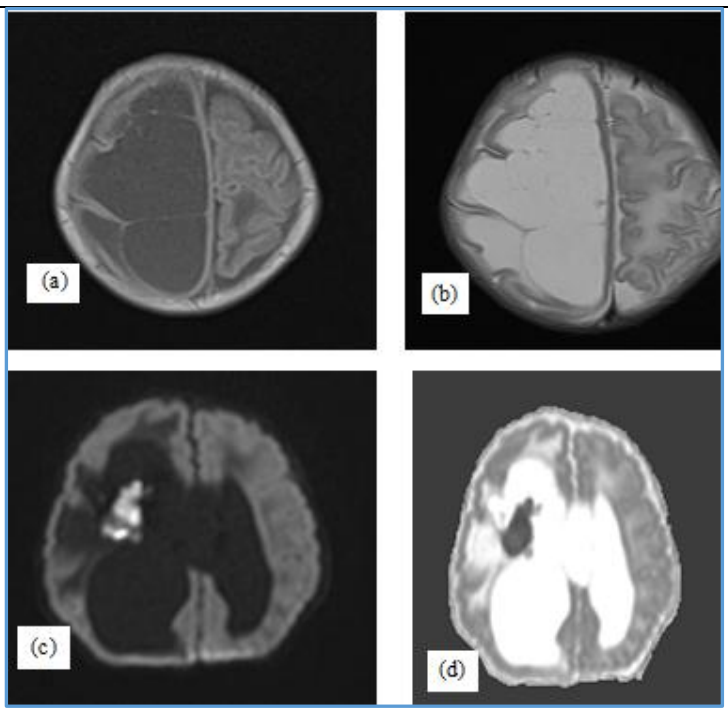

Figure: (a) Axial FLAIR, (b) Axial T2W images showing extensive area of cystic encephalomalacia following CSF signal involving right cerebral hemisphere (c) Axial DWI and (d) Axial ADC showing diffusion restriction in the area of dilated right lateral ventricle s/o Acute infarction
Case 3

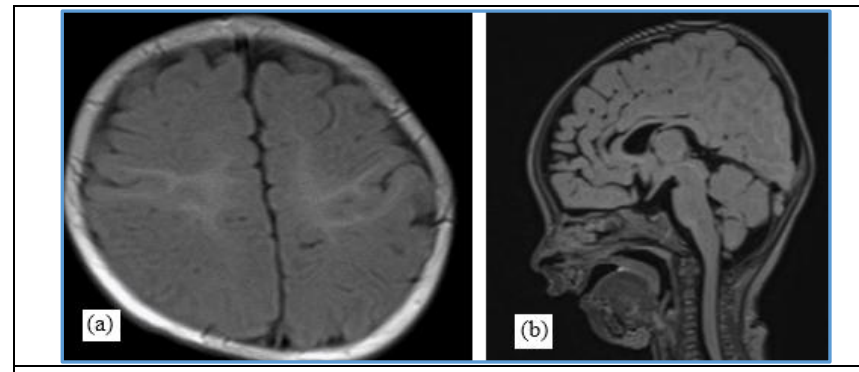

Figure (a) Axial T1W image in patient with history of birth asphyxia, showing hyperintensities in the peri-Rolandic areas in both the cerebral hemispheres. (b) Sagittal FLAIR MR images showing corpus callosum thinning at body level.

Case 4
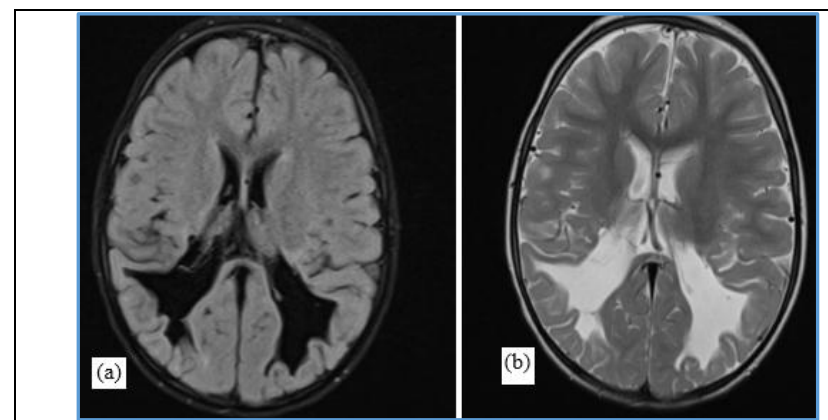

Figure (a) Axial FLAIR, (b) Axial T2 MR images at the ventricular level showing loss of white matter in occipital lobe with enlargement of posterior horns of bilateral lateral ventricles suggestive of periventricular leukomalacia.

Case 5
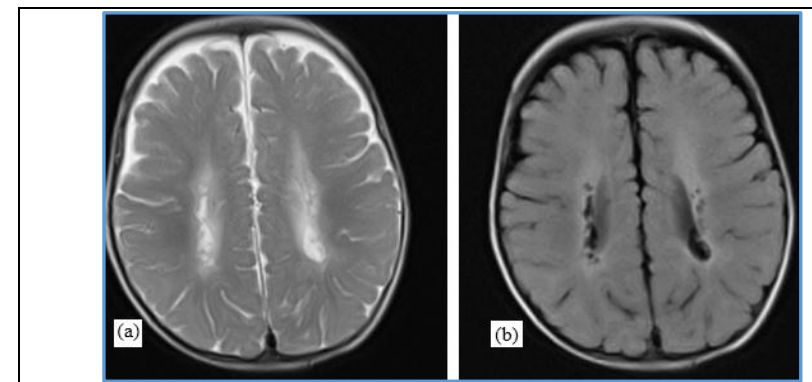

Figure (a) Axial T2WI, (b) Axial FLAIR MR images of a $31 / 2$-year-old child with cerebral palsy, born preterm with low birth weight, at the level of lateral ventricles show reduction in volume and gliosis of the periventricular white matter with mild dilatation and wavy margins of the lateral ventricles.

\section{Case 6}
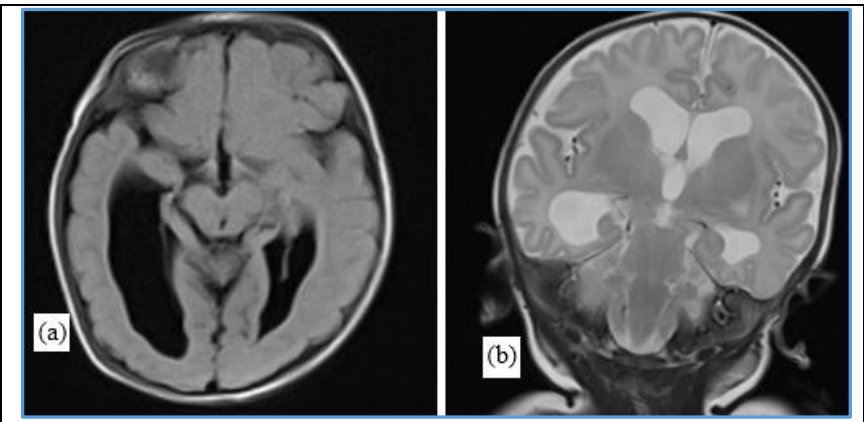

Figure (a) Axial FLAIR, (b) Coronal T2 MR images showing significant loss of white matter in bilateral temporal lobes with dilatation of lateral ventricles (right > left) suggestive of periventricular leukomalacia 
Case 7
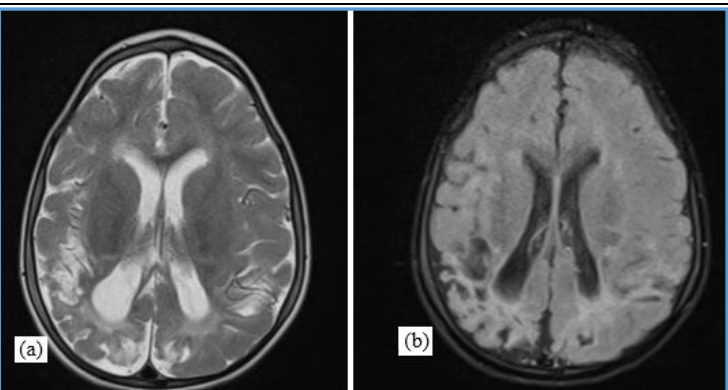

Figure (a) Axial T2WI, (b) Axial FLAIR MR images at the level of body of lateral ventricles showing bilateral periventricular hyperintensities with reduced white matter, subcortical white matter cystic changes and gliosis in both parietal lobes with mild ex vacuo dilatation of the b/l atria

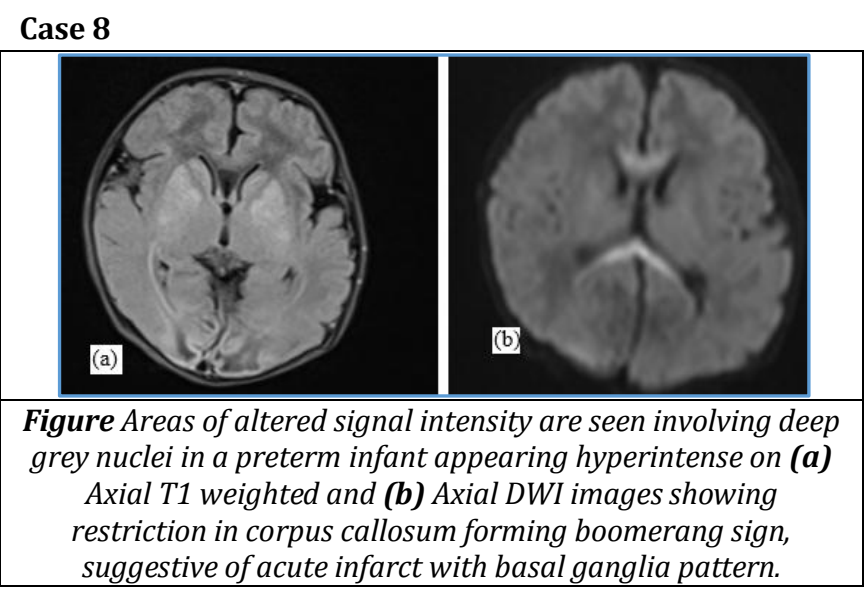

Case 9
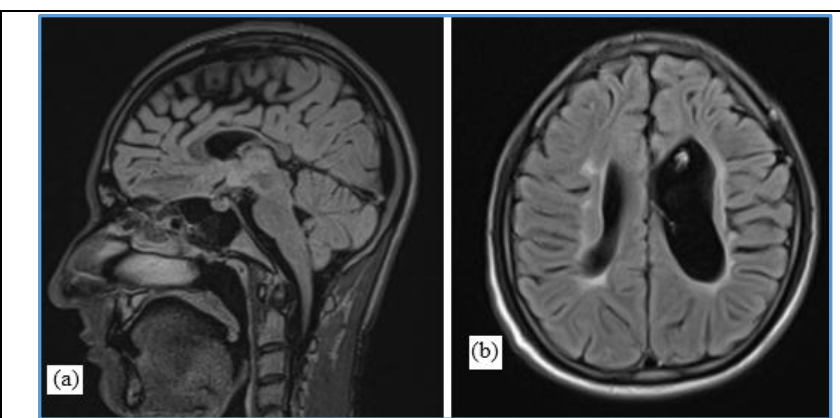

Figure (a) Sagittal and (b) Axial FLAIR MR images showing corpus callosum thinning, significant loss of periventricular white matter with dilatation of lateral ventricles (left > right) and periventricular hyperintense signals.

\section{CONCLUSION}

MRI being the most effective method for detection of the lesions of the hypoxic-ischemic injury, is now often used for the CNS diagnostics in preterm neonates, neonates with very low birth weight, and term neonates with significant perinatal history. Correct radiological diagnosis can truly contribute to the efficient implementation of care over the sick child. Advancements in MRI like DTI can be useful for detailed evaluation of patients with HIE with inconclusive study on conventional MRI brain studies. In our study, MRI was able to differentiate between various patterns of brain injury, depending on the brain maturity, severity and duration of the ischemic insult. We observed that most of the patients suffering from HIE presented in the late stages. This suggests that there is a lack of public health awareness as well as neonatologists in developing countries. Public health awareness needs to be improved. Regular antenatal check-up and timely referral to appropriate centres should be encouraged. Regular follow up MRI studies for the affected children is extremely important to know the prognosis of the condition

\section{REFERENCES}

[1] Allen KA, Brandon DH. Hypoxic ischemic encephalopathy: pathophysiology and experimental treatments. Newborn and Infant Nursing Reviews 2011;11(3):125-33.

[2] Varghese B, Xavier R, Manoj VC, et al. Magnetic resonance imaging spectrum of perinatal hypoxicischemic brain injury. The Indian Journal of Radiology \& Imaging 2016;26(3):316-27.

[3] Qureshi AM, Ur Rehman A, Siddiqi TS. Hypoxic ischemic encephalopathy in neonates. J Ayub Med Coll Abbottabad 2010;22(4):190-3.

[4] Millar LJ, Shi L, Hoerder-Suabedissen A, et al. Neonatal hypoxia ischaemia: mechanisms, models and therapeutic challenges. Frontiers in Cellular Neuroscience 2017;11:78.

[5] Bano S, Chaudhary V, Garga UC. Neonatal hypoxicischemic encephalopathy: a radiological review. Journal of Pediatric Neurosciences 2017;12(1):1-6.

[6] Cabaj A, Bekiesińska-Figatowska M, Mądzik J. MRI patterns of hypoxic-ischemic brain injury in preterm and full term infants-classical and less common MR findings. Polish Journal of Radiology 2012;77(3):71-6.

[7] Shroff MM, Soares-Fernandes JP, Whyte H, et al. MRI for diagnostic evaluation of encephalopathy in the newborn. Radiographics 2010;30(3):763-80.

[8] Haller S, Kövari E, Herrmann FR, et al. Do brain T2/FLAIR white matter hyperintensities correspond to myelin loss in normal aging? A radiologicneuropathologic correlation study. Acta Neuropathologica Communications 2013;1(1):14.

[9] Barkovich AJ. The encephalopathic neonate: choosing the proper imaging technique. Am J Neuroradiol 1997;18(10):1816-20.

[10] Heinz ER, Provenzale JM. Imaging findings in neonatal hypoxia: a practical review. Am J Roentgenol 2009;192(1):41-7.

[11] Lakhkar BN, Aggarwal M, John JR. MRI in white matter diseases - clinico-radiological correlation. Indian J Radiol Imaging 2002;12(1):43-50.

[12] Connolly DJ, Widjaja E, Griffiths PD. Involvement of the anterior lobe of the cerebellar vermis in perinatal profound hypoxia. American Journal of Neuroradiology 2007;28(1):16-9.

[13] Yin R, Reddihough D, Ditchfield M, et al. Magnetic resonance imaging findings in cerebral palsy. J Paediatr Child Health 2000;36(2):139-44.

[14] Khaladkar SM, Gujarathi AM, Kamal V, et al. MRI Brain in perinatal hypoxia-a case series. IOSR J Dent \& Med Sci (IOSR-JDMS) 2016;15(7):100-14.

[15] Rutherford M, Pennock J, Schwieso J, et al. Hypoxicischaemic encephalopathy: early and late magnetic resonance imaging findings in relation to outcome. Arch Dis Child Fetal Neonatal Ed 1996;75(3):F145-51.

[16] Serdaroglu G, Tekgul H, Kitis O, et al. Correlative value of magnetic resonance imaging for neuro-developmental outcome in periventricular leukomalacia. Dev Med Child Neurol 2004;46(11):733-9.

[17] Truwit CL, Barkovich AJ, Koch TK, et al. Cerebral palsy: MR findings in 40 patients. American Journal of Neuroradiology 1992;13(1):67-78. 